\title{
Perfil epidemiológico dos indivíduos HIV positivo e coinfecção HIV-Leishmania em um serviço de referência em São Luís, MA, Brasil
}

\author{
The epidemiological profile of HIV-positive individuals \\ and HIV-Leishmaniasis co-infection in a referral center \\ in São Luis, Maranhão, Brazil
}

Flávia Lopes Carvalho ${ }^{1}$

Diana Leite Sousa Aires ${ }^{2}$

Zeyle Fernandes Segunda ${ }^{3}$

Conceição Maria Pedrozo e Silva de Azevedo ${ }^{4}$

Rita da Graça Carvalhal Frazão Corrêa ${ }^{5}$

Dorlene Maria Cardoso de Aquino ${ }^{5}$

Arlene de Jesus Mendes Caldas ${ }^{5}$

${ }^{1}$ Programa de Pós-

Graduação em Saúde Materno Infantil,

Universidade Federal do

Maranhão (UFMA). R.

Viana Vaz no 230, Centro.

65020-660 São Luís

Maranhão.

ritacarvalhal@hotmail.com

${ }^{2}$ Secretaria de Saúde, Estado

do Maranhão.

${ }^{3}$ Hospital Universitário,

UFMA.

${ }^{4}$ Departamento de

Medicina, UFMA.

${ }^{5}$ Departamento de

Enfermagem, UFMA.

\begin{abstract}
AIDS is a public health problem and visceral leishmaniasis (VL) is the most frequent co-infection. This study seeks to describe the epidemiological aspects of HIV-positive individuals and to investigate the occurrence of HIV-Leishmania co-infection. It is a descriptive study with $287 \mathrm{HIV}=$ positive individuals assisted at the reference center for HIV/AIDS in São Luís, MA. Montenegro's intradermal reaction and bone marrow aspiration were performed for detecting the Leishmania sp. infection. Epidemiological data were collected by means of a questionnaire. The chi-square test was used for gender differences, with $p<0.05$ of statistical significance level. There was statistical significance among men regarding the use of septic tanks, and income of up to two salaries. Regarding exposure, heterosexual orientation was the most frequent category for both genders, and we observed an increase in this category among women. Among HIV-positive individuals, $4.2 \%$ had suggestive symptoms of VL with identification of Leishmania amastigotes in bone marrow aspirate (myelogram). The profile of HIV-positive individuals did not differ from what is commonly found in Brazil, with predominance of young people of productive age. Furthermore, a superposition of the transmission areas for HIV-LV co-infection was found.
\end{abstract}

Key words HIV/Aids, Visceral leshmaniasis, Coinfection
Resumo A Aidsé um problema de saúde pública e a Leishmaniose visceral (LV) a forma mais frequente de coinfecção. O objetivo é descrever aspectos epidemiológicos dos indivíduos HIV positivo e investigar a ocorrência de coinfecção HIV-Leishmania. Trata-se de estudo descritivo realizado com 287 indivíduos HIV positivo atendidos no Centro de Referência para DST/Aids em São Luís (MA). Foi realizado teste de Intradermorreação de Montenegro, aspirado de medula óssea para detecção da infecção por Leishmania sp e aplicado um questionário na coleta dos dados epidemiológicos. $O$ teste qui-quadrado foi usado para diferenças entre gênero, com $p<0,05$ de significância. Houve significância estatística entre os homens relacionados ao uso de fossa séptica e renda até dois salários. Quanto à exposição, foi predominante a categoria heterossexual para ambos os sexos, sendo observado um aumento entre as mulheres. Entre os indivíduos HIV positivo 4,2\% apresentavam sintomatologia sugestiva de LV sendo identificadas formas amastigotas para Leishmania sp no aspirado de medula óssea. O perfil dos indivíduos HIV positivo não diferiu de outras regiões do Brasil com predominio de jovens em idade produtiva, sendo constatada uma sobreposição das áreas de transmissão para a coinfecção HIV-LV.

Palavras-chave HIV/Aids, Leishmaniose visceral, Coinfecção 


\section{Introdução}

A síndrome de imunodeficiência adquirida (Aids) é considerada um grande problema de saúde pública. Nos últimos tempos, poucos agravos à saúde geraram tamanho grau de interesse dos profissionais de saúde, de atividade científica, de estigma e de preconceito como a Aids. O número de pessoas infectadas pelo vírus da imunodeficiência humana (HIV), o modo de transmissão e o impacto gerado na sociedade caracterizou a Aids como uma doença de grande dimensão social ${ }^{1,2}$.

$\mathrm{Na}$ América Latina, 1,7 milhões de pessoas estão infectadas pelo HIV. A prevalência de infecções no Brasil é estimada em 630.000, com aproximadamente 34.500 novos casos de Aids por ano. Desde os primeiros casos da doença no país até junho de 2009, foram registrados 544.849 casos e 217.091 óbitos por Aids 3 .

A Aids tem aumentado entre as mulheres e a razão de casos homens/mulheres cada vez fica mais próxima. A expansão da Aids entre as mulheres vem sendo observada em quase todos os continentes, e estas representam $48 \%$ dos casos no mundo. No Brasil, a razão entre os sexos mostra claramente esta tendência: em 1986, eram 15,1 casos masculinos para um feminino; em 1996 eram 2,6; e, em 2009, 1,6 casos em homens para um caso em mulheres.

No Maranhão foram notificados 4.132 casos de Aids, no período de 1985 até janeiro de 2009 com incidência de 5,5 casos por 100.000 habitantes. Em 2009 no município de São Luís foram notificados 2.607 casos novos correspondendo a 63,1\% no Estado do Maranhão, sendo a razão por sexo de 1,9 homens/1,0 mulher ${ }^{4}$.

Com o surgimento da Aids houve o aparecimento de manifestações clínicas atípicas, bem como o surgimento ou ressurgimento de uma doença em decorrência de imunossupressão, caracterizando a presença de doenças oportunistas relacionadas à Aids. Dentre as doenças destacamse o ressurgimento da tuberculose em determinadas regiões onde a doença estava sob controle, assim como apresentações atípicas de doença de Chagas e de Leishmaniose Visceral (LV) ${ }^{5-7}$.

A LV é a forma clínica das Leishmanioses mais associada ao HIV. A coinfecção LV-HIV tem sido considerada como doença emergente em várias regiões do mundo em função da superposição geográfica das duas infecções, como consequência da urbanização das leishmanioses e da ruralização da infecção por HIV. A coinfecção LVHIV tem sido considerada na Europa como um problema de saúde pública onde a proporção de indivíduos com LV e infectados pelo HIV chega a $77 \%{ }^{7,8}$. Estima-se que $10 \%$ dos portadores de HIV apresentem infecção assintomática por Leishmania sp. e destes $2 \%$ a $9 \%$ dos co-infectados desenvolverão LV clinicamente manifesta9.

No Brasil, o primeiro caso de coinfecção Leishmania-HIV foi descrito em 1987, com aumento progressivo de casos nos anos seguintes ${ }^{10}$. As regiões com maior percentual de casos de coinfecção foram o Nordeste e o Sudeste, onde justamente predominam, respectivamente, os casos de LV e Aids².

No Maranhão foram documentados 31 casos de coinfecção LV-HIV no período de 2000 a $2005^{11}$. Os dados no Estado são preocupantes tanto em relação à LV quanto à infecção pelo HIV. A LV foi registrada em 54,8\% dos municípios maranhenses e casos de Aids em 64,1\% $\%^{4}$.

Por se tratar de duas endemias brasileiras, em especial no Estado do Maranhão, estudos relacionados à coinfecção HIV-LV são importantes por retratar o perfil epidemiológico da doença e assim contribuir com estratégias para a saúde das populações e ao mesmo tempo dando ênfase ao cuidado da saúde individual voltada principalmente para a questão do diagnóstico precoce em casos de febre persistente. Este estudo tem como objetivo descrever aspectos epidemiológicos dos indivíduos HIV positivos e investigar a ocorrência de coinfecção HIV-Leishmania.

\section{Métodos}

Realizou-se um estudo descritivo com indivíduos HIV positivo atendidos em um Centro de Referência para DST/Aids em São Luís (MA), no período de março/2006 a dezembro/2008. O Centro de Referência atende pacientes de todo o Estado.

Inicialmente os indivíduos eram informados sobre a importância e o objetivo da pesquisa e ao confirmarem sua participação, assinavam o Termo de Consentimento Livre e Esclarecido. O questionário foi aplicado por profissional previamente treinado, contendo as seguintes variáveis: características sociodemográficas, características epidemiológicas da LV, categorias de exposição e orientação sexual, ocorrência de coinfecção; realização do exame físico e o teste da Intradermorreação de Montenegro (IDRM) para detecção da infecção por Leishmania sp. Os indivíduos com quadro sugestivo de LV (febre prolongada, hepatoesplenomegalia) eram encaminhados para o Hospital de referência para realização do exame parasitológico (identificação de formas amas- 
tigotas para Leishmania sp no aspirado de medula óssea - Mielograma, em esfregaços corados pelo método Giemsa).

Para realização do teste de IDRM, foi utilizado antígeno preparado no Centro de Pesquisas Gonçalo Moniz/Fiocruz, Salvador-BA, com formas amastigotas de L. amazonensis ( $\mathrm{MHOMBr}$ 88-BA-84 125), de acordo com a técnica descrita por Reed et al. ${ }^{12}$. Inoculou-se $0,1 \mathrm{ml}$ do preparado antigênico na face anterior do antebraço, e a leitura foi realizada depois 48 a 72 horas, de acordo com a técnica de Sokal ${ }^{13}$. O teste foi positivo quando um dos diâmetros da enduração foi igual ou superior a $5 \mathrm{~mm}$.

Considerou-se como coinfecção HIV-LV assintomática: indivíduos HIV positivo e IDRM positiva sem sintomas sugestivos de LV (febre prolongada, hepatoesplenomegalia); e coinfecção sintomática: indivíduos HIV positivo com sinais e sintomas sugestivos de LV, e com o diagnóstico parasitológico confirmado por meio da identificação de formas amastigotas para Leishmania sp no aspirado de medula óssea (Mielograma) em esfregaços corados pelo método Giemsa.

Os dados foram digitados e analisados no Programa EPI-INFO, versão 3.4.3 (CDC-Atlanta-EUA). O teste qui-quadrado foi utilizado para verificar as diferenças entre sexos. Quando o valor de $p>0,05$ considerou-se a diferença estatisticamente significante.

O estudo foi aprovado pelo Comitê de Ética em Pesquisa do Hospital Universitário da Universidade Federal do Maranhão (CEP-HUUFMA).

\section{Resultados}

Participaram do estudo 287 indivíduos HIV positivo. Quanto ao sexo observou-se que 46,3\% eram mulheres e $53,7 \%$ homens, correspondendo a uma proporção de 1:1 mulheres para 1:5 homens, com predominância a faixa etária entre 18 a 40 anos em ambos os sexos. A localização, tipo de casa, destino do lixo e origem da água não apresentaram diferenças estatisticamente significante entre homens e mulheres. Por outro lado, entre os homens houve predominância do uso da rede de esgoto e fossa séptica. Quanto à situação conjugal e escolaridade houve predominância de indivíduos sem companheiro(a) e o ensino médio completo foi predominante em ambos os sexos $(p=0,003)$. A ocupação de dona de casa e doméstica foi predominante entre as mulheres e a maioria dos homens era de aposentados. Entre as mulheres a renda familiar era menor que 1 salário mínimo $(55,6 \%)$ e entre os homens o salário mínimo era de 1 a 2 salários mínimo $(55,8 \%)$ (Tabela 1$)$.

Analisando as características epidemiológicas, observou-se que não houve diferença estatisticamente significante entre gêneros. Entretanto, a presença de chiqueiro, galinheiro $(\mathrm{p}<0,006) \mathrm{e}$ local próximo à residência $(\mathrm{p}<0,001)$ estavam mais presentes nas moradias das mulheres ( $\mathrm{Ta}-$ bela 2).

Quanto à categoria de exposição, houve predominância da categoria heterossexual para ambos os sexos, porém foi observado um aumento nesta categoria entre as mulheres $(\mathrm{p}<0,001)$ (Tabela 3).

Dos 287 indivíduos HIV positivo que realizaram IDRM somente em 271 foi realizada a leitura; destes, $1,5 \%$ apresentaram positividade ao teste. Houve uma perda de 1,5\% devido ao não comparecimento dos indivíduos para a realização da leitura do teste (Tabela 4).

Em 12 (4,2\%) indivíduos HIV positivo e com sintomatologia sugestiva de LV foram identificadas formas amastigotas para Leishmania sp no aspirado de medula óssea (Mielograma). Destes, 09 eram do sexo masculino e 03 feminino, na faixa etária entre 18 a 40 anos. Vale ressaltar que foram excluídos os indivíduos com IDRM positiva no cálculo do percentual com mielograma positivo.

\section{Discussão}

As recentes modificações nos perfis epidemiológicos da Aids e da LV no Brasil, resultante da interiorização da infecção pelo HIV simultânea à urbanização da LV, bem como o aumento do número de casos em mulheres, apontam para maior exposição da população às duas infecções.

Embora a predominância do sexo masculino caracterize o padrão encontrado para casos de Aids no Brasil e no Estado do Maranhão (1,2 homens para uma mulher $)^{2-4}$, dados epidemiológicos vêm demonstrando a aproximação da relação dos casos de Aids entre homens e mulheres refletindo o fenômeno chamado de feminização da Aids, resultado do aumento da transmissão heterossexual do HIV, decorrente do comportamento sociossexual da população, associado aos aspectos de vulnerabilidade biológica da mulher ${ }^{14,15}$.

A média de idade foi 36,5 anos, predominando a faixa etária entre 18 a 40 anos, demonstrando serem adultos jovens, corroborando com os 
Tabela 1. Características sociodemográficas por sexo dos indivíduos HIV positivo. São Luís (MA), 2009.

\begin{tabular}{|c|c|c|c|c|c|c|}
\hline \multirow{3}{*}{ Característica } & \multicolumn{4}{|c|}{ Sexo } & \multirow[b]{3}{*}{$\chi^{2}$} & \multirow[b]{3}{*}{$p$} \\
\hline & \multicolumn{2}{|c|}{ Feminino } & \multicolumn{2}{|c|}{ Masculino } & & \\
\hline & $\mathbf{n}$ & $\%$ & $\mathbf{n}$ & $\%$ & & \\
\hline Idade (anos) & & & & & 3,881 & 0,144 \\
\hline $18-40$ & 86 & 64,7 & 100 & 64,9 & & \\
\hline $41-60$ & 40 & 30,1 & 52 & 33,8 & & \\
\hline$>60$ & 7 & 5,2 & 2 & 1,3 & & \\
\hline Cor (referida) & & & & & 8,514 & 0,014 \\
\hline Branca & 11 & 8,3 & 30 & 19,5 & & \\
\hline Negra & 13 & 9,7 & 19 & 12,3 & & \\
\hline Parda & 109 & 82,0 & 105 & 68,2 & & \\
\hline Moradia & & & & & 1,685 & 0,194 \\
\hline Tijolo & 122 & 91,6 & 147 & 95,5 & & \\
\hline Taipa & 11 & 8,4 & 7 & 4,5 & & \\
\hline Destino dos dejetos & & & & & 11,532 & 0,003 \\
\hline Rede de esgoto & 89 & 66,9 & 106 & 68,8 & & \\
\hline Fossa séptica & 21 & 15,8 & 39 & 25,3 & & \\
\hline Fossa negra & 23 & 17,3 & 9 & 5,7 & & \\
\hline Destino do lixo & & & & & 0,473 & 0,491 \\
\hline Coleta pública & 120 & 90,2 & 135 & 87,7 & & \\
\hline Queimada & 13 & 9,8 & 19 & 12,3 & & \\
\hline Origem da agua & & & & & 1,892 & 0,169 \\
\hline Rede pública & 114 & 85,8 & 140 & 90,9 & & \\
\hline Poço artesiano & 19 & 14,2 & 14 & 9,1 & & \\
\hline Moradia (zona) & & & & & 0,060 & 0,806 \\
\hline Urbana & 122 & 91,7 & 140 & 90,9 & & \\
\hline Rural & 11 & 8,3 & 14 & 9,1 & & \\
\hline Situação conjugal & & & & & 0,227 & 0,634 \\
\hline Com companheiro/a & 52 & 39,0 & 56 & 36,4 & & \\
\hline Sem companheiro/a & 81 & 61,0 & 98 & 63,6 & & \\
\hline Escolaridade & & & & & 11,286 & 0,080 \\
\hline Analfabeto & 7 & 5,3 & 4 & 2,6 & & \\
\hline Fundamental Incompleto & 38 & 28,6 & 36 & 23,4 & & \\
\hline Fundamental completo & 15 & 11,3 & 12 & 7,8 & & \\
\hline Ensino Médio Incompleto & 18 & 13,5 & 25 & 16,2 & & \\
\hline Ensino Médio Completo & 54 & 40,6 & 65 & 42,2 & & \\
\hline Ensino Superior Incompleto & 1 & 0,7 & 9 & 5,8 & & \\
\hline Ensino Superior Completo & - & - & 3 & 2,0 & & \\
\hline Ocupação & & & & & 130,329 & $<0,001$ \\
\hline Dona de casa & 51 & 38,2 & - & - & & \\
\hline Doméstica & 28 & 21,1 & - & - & & \\
\hline Vendedor & 10 & 7,5 & 16 & 10,4 & & \\
\hline Estudante & 5 & 3,8 & 12 & 7,8 & & \\
\hline Aposentado & 6 & 4,6 & 26 & 16,0 & & \\
\hline Autônomo & 33 & 24,8 & 100 & 64,9 & & \\
\hline Renda familiar (salário mínimo) & & & & & 12,228 & 0,002 \\
\hline$<1$ & 74 & 55,6 & 60 & 39,0 & & \\
\hline 1 a 2 & 47 & 35,4 & 86 & 55,8 & & \\
\hline$>2$ & 12 & 9,0 & 8 & 5,2 & & \\
\hline Total & 133 & 100,0 & 154 & 100,0 & & \\
\hline
\end{tabular}

$\chi^{2}=$ Qui-quadrado 
Tabela 2. Características epidemiológicas da Leishmaniose visceral (LV) por gênero dos indivíduos HIV positivo. São Luís (MA), 2009.

\begin{tabular}{|c|c|c|c|c|c|c|}
\hline \multirow{3}{*}{ Característica } & \multicolumn{4}{|c|}{ Gênero } & \multirow[b]{3}{*}{$\chi^{2}$} & \multirow[b]{3}{*}{$p$} \\
\hline & \multicolumn{2}{|c|}{ Feminino } & \multicolumn{2}{|c|}{ Masculino } & & \\
\hline & $\mathbf{n}$ & $\%$ & $\mathbf{n}$ & $\%$ & & \\
\hline LV na família & & & & & 0,024 & 0,988 \\
\hline Sim & 4 & 2,3 & 5 & 3,2 & & \\
\hline Não & 128 & 91,7 & 148 & 96,1 & & \\
\hline Não sabe informar & 1 & 0,8 & 1 & 0,7 & & \\
\hline LV na vizinhança & & & & & 2,514 & 0,285 \\
\hline Sim & 3 & 2,3 & 2 & 1,3 & & \\
\hline Não & 127 & 91,7 & 148 & 96,1 & & \\
\hline Não sabe informar & 8 & 6,0 & 4 & 2,6 & & \\
\hline Chiqueiro & & & & & 7,541 & 0,006 \\
\hline Sim & 21 & 15,8 & 8 & 5,8 & & \\
\hline Não & 112 & 84,2 & 145 & 94,2 & & \\
\hline Criação de cães & & & & & 0,010 & 0,976 \\
\hline Sim & 84 & 63,2 & 97 & 63,0 & & \\
\hline Não & 49 & 36,8 & 57 & 37,0 & & \\
\hline Galinheiro & & & & & 6,203 & 0,013 \\
\hline Sim & 44 & 33,1 & 31 & 20,1 & & \\
\hline Não & 89 & 66,9 & 123 & 79,9 & & \\
\hline Local da residência & & & & & 20,684 & 0,001 \\
\hline Terreno alagado & 19 & 14,3 & 5 & 3,2 & & \\
\hline Esgoto a céu aberto & 35 & 26,3 & 28 & 18,2 & & \\
\hline Rio e lago & 4 & 3,0 & 15 & 9,7 & & \\
\hline Lixeiro & 12 & 9,0 & 18 & 11,7 & & \\
\hline Mata & 60 & 45,1 & 79 & 51,3 & & \\
\hline Praças & 3 & 2,3 & 9 & 5,8 & & \\
\hline Presença de flebótomo & & & & & 3,029 & 0,220 \\
\hline Sim & 15 & 11,3 & 16 & 10,4 & & \\
\hline Não & 89 & 66,9 & 116 & 75,3 & & \\
\hline Não sabe informar & 29 & 21,8 & 22 & 14,3 & & \\
\hline Total & 133 & 100,0 & 154 & 100,0 & & \\
\hline
\end{tabular}

$\chi^{2}=$ Qui-quadrado

achados de Rabello et al. ${ }^{10}$. Destes, $46,2 \%$ eram mulheres em idade reprodutiva. Por outro lado, observou-se um crescimento da faixa etária que compreende as pessoas mais velhas, demonstrando tendência no crescimento da taxa de incidência em homens e mulheres a partir dos 41 anos. Quanto aos indivíduos maiores de 60 anos, os resultados mostraram uma taxa superior à nacional $(2,4 \%)$ encontrada no período de 1980 a junho de $2008^{14}$.

Os participantes em sua maioria residiam sem companheiro e em zona urbana com acesso ao saneamento básico, características que não interferem para a aquisição do vírus do HIV, e não são características comumente encontradas em ambientes que favorecem a $\mathrm{LV}^{14,16}$, mas que podem constituir um indicador importante para avaliar a qualidade de vida da população bem como a expansão das duas patologias. Esses resultados são expressivos e demonstram outra faceta da epidemia, que é a condição solitária dos indivíduos com Aids.

O percentual de indivíduos HIV positivo com ensino fundamental ou ensino médio completo ou incompleto entre os homens foi superior em relação às mulheres, o que pode ter influenciado no tipo de ocupação, pois a maioria das mulheres realizava "afazeres domésticos", revelando que a Aids vem afetando progressivamente, indivíduos em posições desvantajosas em relação ao merca- 


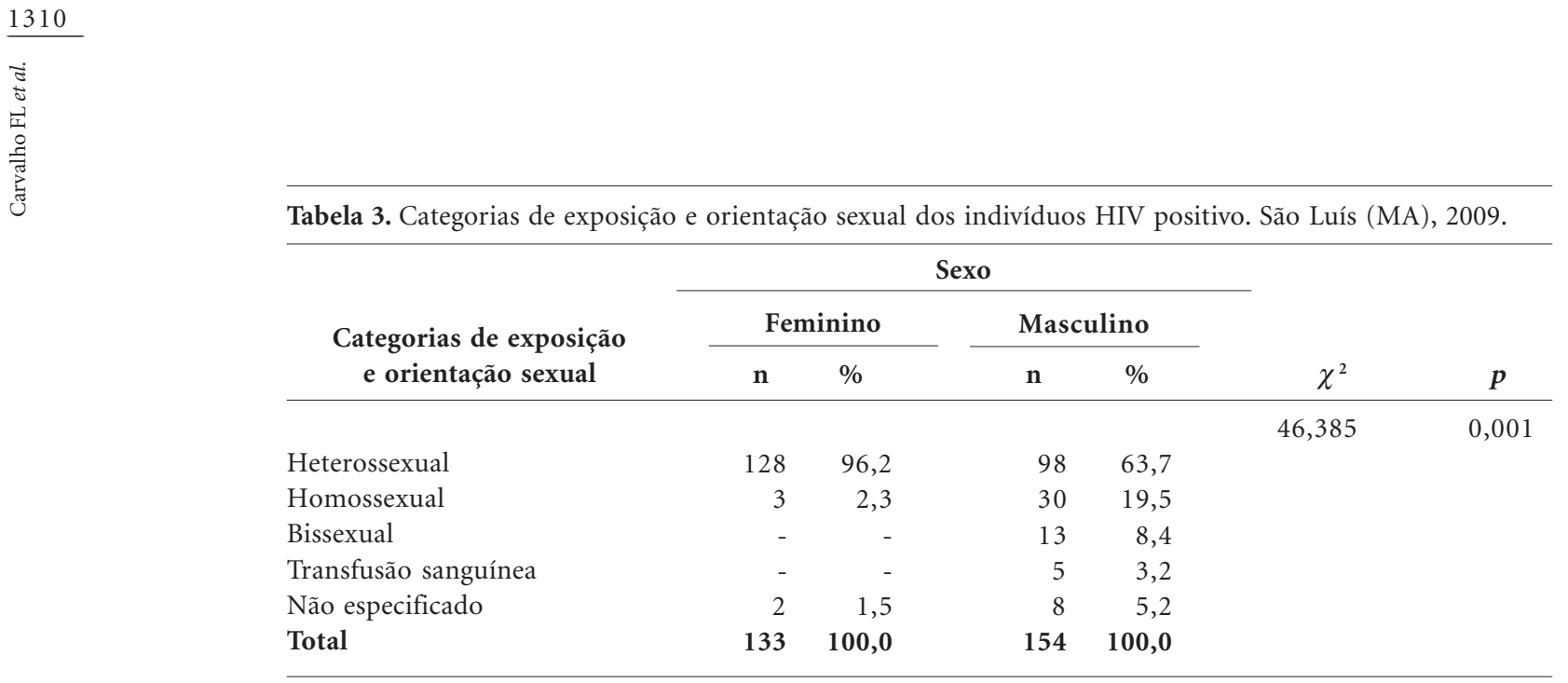

$\chi^{2}=$ Qui-quadrado

Tabela 4. Ocorrência de co-infecção Leishmanial HIV em indivíduos HIV positivo. São Luís (MA), 2009.

\begin{tabular}{|c|c|c|}
\hline Teste & $\mathbf{n}(\mathbf{N})$ & $\%$ \\
\hline $\begin{array}{l}\text { Intradermorreação de } \\
\text { Montenegro (IDRM)* }\end{array}$ & $4(271)$ & 1,5 \\
\hline Mielograma & $12(283)$ & 4,2 \\
\hline
\end{tabular}

* enduração $\geq 5 \mathrm{~mm}$

do de trabalho. Fato este, também confirmada pela renda familiar de ambos os sexos que era até dois salários mínimos. Estudos realizados por Fide e Mode $^{17}$, na Filadélfia (Estados Unidos) e por Szwarcwald et al. ${ }^{18}$ no Rio de Janeiro, mostrou o crescimento da epidemia do HIV em status sociais menos favorecidos e com baixa escolaridade afetando mais intensamente as mulheres.

Neste estudo a maioria da população feminina contraiu a infecção através de contatos heterossexuais, concordando com os achados de Galvão et al., ${ }^{19}$ e Figueiredo et al., ${ }^{20}$ onde a maioria das mulheres foram infectadas por relações heterossexuais.

Não houve registro de casos de LV na família e vizinhança. Esse resultado pode estar relacionado à residência em zona urbana e com saneamento básico. A LV, embora seja uma doença predominante de área rural, nas últimas décadas tem-se estabelecido em áreas urbanas e/ou periurbanas de cidades de médio e grande porte do Nordeste, Sudeste e Centro-Oeste, tais como: São Luís (MA), Teresina (PI), Belo Horizonte (MG), Campo Grande (MS) ${ }^{14,16}$.
Os resultados relacionados à coinfecção HIVLV deste estudo foram ligeiramente superiores ao encontrado no Brasil (3,7\%) entre 2007 e 2008. No Maranhão, no mesmo período foram registrados 30 casos de coinfecção ${ }^{2}$. É um percentual relativamente alto, considerando o tamanho da amostra estudada, porém similar aos estudos realizados na Espanha por Delgado et al. ${ }^{21}$ que foi de $4,2 \%$, e Reus et al. ${ }^{22}$ de $4,3 \%$, e diferente do resultado encontrado na Etiópia, que foi de 15,0 a $30,0 \%{ }^{23}$.

Como a IDRM avalia a resposta celular e os pacientes imunodeprimidos não apresentam positividade ao teste, acredita-se que os indivíduos que apresentaram positividade ao teste apresentaram boa resposta celular, sendo classificados, portanto, na categoria de forma assintomática da LV.

Destaca-se como importante o fato dos indivíduos estarem vinculados a serviço de referência, com acesso aos atendimentos e garantia de medicamentos representando fator que contribuiu para a diminuição das perdas.

O perfil dos indivíduos HIV positivo não diferiu do padrão encontrado no Brasil, com predomínio de jovens em idade produtiva, porém foi constatada uma sobreposição das áreas de transmissão das duas patologias, possibilitando o aparecimento da coinfecção HIV-LV.

Como a LV representa uma infecção oportunista sugere-se a realização de estudos sobre diagnóstico diferencial de todo indivíduo, não só HIV positivo, com febre persistente, mas também em todo imunodeprimido procedente de área endêmica.

É importante destacar a atenção dos profissionais de saúde, particularmente daqueles que trabalham em áreas endêmicas de leishmaniose, 
para a ocorrência de casos de coinfecção LV-HIV considerando o crescimento do número de casos à medida que a Aids se expande para as pequenas localidades e as zonas rurais do país, dando ênfase ao cuidado da saúde individual para a questão do diagnóstico precoce em casos de febre persistente.

\section{Colaboradores}

FL Carvalho participou da coleta de dados e da elaboração do artigo, DLS Aires e ZF Segunda participaram da coleta de dados, CMPS de Azevedo participou do atendimento aos pacientes e elaboração do artigo, RGCF Corrêa e DMC Aquino participaram da elaboração do artigo e AJM Caldas coordenou e participou em todas as etapas do estudo e elaboração do artigo

\section{Agradecimentos}

Agradecemos aos profissionais do Centro de Referência para DST/Aids em São Luís (MA) e a Fundação de Amparo a Pesquisa e ao Desenvolvimento Científico e Tecnológico do Maranhão (FAPEMA) pelas bolsas concedidas aos alunos. 


\section{Referências}

1. Alves MTSSB, Silva AAM, Nemes MIB, Brito LGO. Tendência da incidência e da mortalidade por Aids no Maranhão, 1985 a 1998. Rev Saude Publica 2003; 37(2):177-182.

2. Sousa-Gomes ML, Maia-Elkhoury ANS, Pelissari DM, Lima Junior FEF, Sena JM, Cechinel MP. Coinfecção Leishmania-HIV no Brasil: aspectos epidemiológicos, clínicos e laboratoriais. Epidemiol. Serv. Saúde 2011; 20(4):519-526.

3. Brasil. Ministério da Saúde (MS). Departamento de DST, Aids e Hepatites Virais. AIDS/DST 2010. Boletim Epidemiológico 2010; 8(1):3-24.

4. Maranhão. Secretaria de Estado da Saúde. Secretaria Adjunta Assistência à saúde. Boletim Epidemiológico/SINAN-Programa Estadual de DST/Aids. São Luís: Secretaria de Estado da Saúde; 2009.

5. Berman JD. Human Leishmaniasis: clinical, diagnostic and chemotherapeutic developments in the last 10 years. Clin Infect Dis. 2005; 24(4):684-703.

6. Lambert N, Mehta B, Walters R, Eron JJ. Chagasic encephalitis as the initial manifestation of AIDS. Ann Intern Med 2006; 144(12):941-943.

7. Desjeux P, Alvar J. Leishmania/HIV co-infections: epidemiology in Europe. Ann Trop Med Parasitol 2003; 97(Supl. 1):3-15.

8. Cruz I, Nieto J, Moreno J, Canavate C, Desjeux P, Alvar J. Leishmania/HIV co-infections in the second decade. Indian J Med Rev 2006; 123(3):357-388.

9. Pintado V, Lopez-Velez R. Visceral leishmaniasis associated with human immunodeficiency virus infection. Enferm Infecc Microbiol Clin 2001; 19(7):353-357.

10. Rabello A, Orsini M, Disch J. Leishmania/HIV coinfection in Brazil: an appraisal. Ann Trop Med Parasitol. 2003; 97(Supl. 1):17-28.

11. Privado W. L. Leishmaniose visceral no estado do Maranhão: co-infecção leishmania-HIV [dissertação]. São Luís: Universidade Federal do Maranhão; 2005.

12. Reed SG, Badaró R, Masur H, Carvalho EM, Lorenço R, Lisboa A, Teixeira R, Johnson Junior WD, Jones TC. Selection of a skin test antigen for American visceral leishmaniasis. A J Tropical Med Hy 1986; 35(1):79-85.

13. Sokal J E. Measurement of delayed skin test responses. N Engl J Med 1975; 293(10):501-502.

14. Brasil. Ministério da Saúde (MS). Manual de Vigilância e Controle da Leishmaniose Visceral. Secretaria de Vigilância em Saúde (SVS). Departamento de Vigilância Epidemiológica. Brasília: MS; 2008.
15. Vieira EM, Villela WV, Réa MF, Fernandes MEL, Franco E, Ribeiro G. Alguns aspectos do comportamento sexual e prática de sexo seguro em homens do Município de São Paulo. Cad Saud Publica 2000; 16(4):997-1009.

16. Caldas AJM, Silva DRC, Pereira CCR, Nunes PMS, Silva BP, Silva AAM. Infecção por Leishmania (leishmania) chagasi em crianças de área endêmica de Leishmaniose visceral americana na ilha de São LuísMA. Rev Soc Bras Med Trop. 2001; 34(5):445-450.

17. Fide D, Mode C. Aids prevalence by income group in Philadelphia. J Acquired Imm Def Syndromes 1992; 5(11):1111-1115.

18. Szwarcwald CL, Bastos FI, Barcellos C, Esteves MAP, Andrade CLT. A disseminação da epidemia da Aids no Brasil, no período de 1987-1996: uma análise espacial. Cad Saude Publica 2000; 16(Supl. 1):7-19.

19. Galvão MTG, Cerqueira ATA, Marcondes-Machado J. Aplicação da qualidade de vida de mulheres com HIV/Aids através do HAT-Qol. Cad Saude Publica 2004; 20(2):430-437.

20. Figueiredo JFC, Reis VMF, Machado AA, SR Oyama, Martinez R, Figueiredo LTM, Fonseca BAL, Costa JC, Moya MJ, Castro G. Características clínicas e epidemiológicas de pacientes da região de Ribeirão Preto, SP, Brasil, com Aids e infecções oportunistas. Medicina 2000; 33:141-114.

21. Delgado FM, Garcia O M, Martos P F, Reguera IJ,Jimenez OF, Colmenero CJ. The clinical and evolutional characteristics of visceral leishmaniasis in patients with HIV infection. An Med Int. 1997; 14(10):506-510.

22. Reus S, Sánchez R, Portilla J. Visceral leishmaniasis: comparative study in patients with and without HIV infection. Enferm Infecc Microbiol Clin 1999; 17(10):515-520.

23. Lyons S, Veeken H, Long J. Visceral leishmaniasis and HIV in Tigray, Ethiopia. Trop Med Int Health. 2003; 8(8):733-739.

Artigo apresentado em 10/05/2012

Aprovado em 30/07/2012

Versão final apresentada em 22/08/2012 